\title{
Genetic Variability and Path Analysis for Quantitative Characters in F4 Generation of Black Gram (Vigna mungo L. Hepper)
}

\author{
Ankur Kumar Singh* and Gaibriyal M. Lal \\ Department of Genetics and Plant Breeding, Sam Higginbottom University of Agriculture, \\ Technology \& Sciences, Prayagraj, Uttar Pradesh, India \\ *Corresponding author
}

A B S T R A C T

\begin{tabular}{|l|}
\hline Ke y w o r d s \\
Black gram, \\
$\begin{array}{l}\text { Genetic variability, } \\
\text { Heritability (\%), } \\
\text { Genetic advance, } \\
\text { Correlation } \\
\text { coefficient, } \\
\text { Path analysis }\end{array}$ \\
\hline Article Info \\
\hline $\begin{array}{l}\text { Accepted: } \\
\text { 04 November } 2020 \\
\text { Available Online: } \\
\text { 10 December } 2020\end{array}$ \\
\hline
\end{tabular}

The experiment consists of 30 genotypes of black gram which was evaluated in a Randomized Block Design with three replications during the zaid season, 2019. Genotype SU-URD-101 was found with high yield along with early flowering. Moderate GCV and PCV were observed fornumber of clusters per plant, harvest index $\%$ and number of pods per plant. High heritability along with moderate gcv was recorded for number of clusters per plant, harvest index $\%$ and number of pods per plant. High genetic advance as $\%$ mean $(>20 \%)$ was recorded for number of clusters per plant, number of pods per plant, and harvest index $\%$ along with high heritability and moderate GCV. Phenotypic and genotypic correlation coefficient analysis revealed that seed yield per plant exhibited positive and significant correlation with harvest index $\%$ only. It exhibited negative and significant correlation with plant days to 50\% flowering and100-seed weight (g). Both at phenotypic and genotypic level, the positive direct effect on grain yield was depicted by harvest index \%, biological yield per plant and number of pods per plant.

\section{Introduction}

Black gram (Vigna mungo (L.) Hepper) popularly known as urd bean or mash, is a grain legume domesticated from $\mathrm{V}$. mungo var. silvestris. It belongs to family Leguminaceae with chromosome number $2 n=2 x=22$. Black gram is reported to be originated in India. Black gram (Vigna mungo (L.) Hepper) is an important grain legume with easily digestible protein and low flatulence contents. It is highly prized pulse, rich in phosphoric acid. Grain of Black gram contains about $25 \%$ protein, $56 \%$ carbohydrate, $2 \%$ fat, $4 \%$ minerals and $0.4 \%$ vitamins. Black gram is 5-10 times more productive than other crops. Besides, being used as food for the cheap source of dietary protein, it is better to use for bean sprouts than mung bean for its longer shelf life. At national level during 2018-19, black gram was grown in 50.31 lakh ha area with 32.84 lakh tonnes of production with average productivity of $655 \mathrm{~kg} / \mathrm{ha}$. In the state of Utter 
Pradesh, it was grown in 5.88 lakh ha area with 3.05 lakh tonnes of production with average productivity of $520 \mathrm{~kg} / \mathrm{ha}$ (Source: Directorate pulse development, Ministry of Agriculture and Farmers Welfare Annual Report (2018-19). Assessment of genetic variability is a basic step in any crop improvement programme. Yield being a complex character, is influenced by a number of yield contributing characters controlled by polygenes and also influenced by the environment. So, the variability in the collections for these characters is the sum total of heredity effects of concerned genes plus the influence of the environment. Hence, it becomes necessary to partition the observed variability into heritable and non-heritable components measured as genotypic and phenotypic coefficients of variation (GCV and PCV), heritability and genetic advance expressed as percent mean.

Seed yield in urd bean is a complex character like other crops, and is determined by various components. Knowledge of genetic variability existing among different parameters is important in crop improvement. Heritability, which measures phenotypic variance and is attributable to genetic causes, is another important consideration for a successful breeding program. Correlation analysis is a biometrical technique to find out the nature and degree of association between various physico-chemical traits indicating yield. However, inheritance of quantitative characters is often influenced by variation in other characters which may be due to pleiotropy or genetic linkage. Hence, knowledge of association between yield and its components obtainable through estimation of genotypic and phenotypic correlations helps a great deal to formulate selection strategies. Path coefficient analysis, on the other hand, is an efficient statistical technique specially designed to quantify the interrelationship of different components and their direct and indirect effects on seed yield. Through this technique yield contributing characters can be ranked and specific traits producing a given correlation can be identified. Hence, the present investigation will be undertaken to characterize the germplasm accessions, to assess the variability and to determine the interrelationship among yield and its contributing characters in black gram.

\section{Materials and Methods}

The present experiment was carried out at the Field Experimentation Centre of Department of Genetics and Plant Breeding, Naini Agricultural Institute, Sam Higginbottom University of Agriculture, Technology and Sciences, Prayagraj, U.P during Zaid-2019. The site of experiment is located at $25.87^{0} \mathrm{~N}$ latitude, $81.51^{0} \mathrm{E}$ longitude and 98 meter above the sea level. The experimental field was divided into 3 blocks of equal size and each row containing single genotype. The spacing of $30 \mathrm{~cm}$ within rows and $10 \mathrm{~cm}$ between the plants was followed. All recommended agronomical cultural practices were carried out to raise a good crop. Observation were recorded based on five randomly selected plants in each genotype in each replication for all important characters viz., plant height $(\mathrm{cm})$, number of primary branches per plant, number of clusters per plant, number of pod per plant, pod length (cm), number of seed per pod, 100seed weight $(\mathrm{g})$, harvest index $(\%)$, biological yield $(\mathrm{g})$ and seed yield per plant (g) except days to $50 \%$ flowering, days to $50 \%$ pod setting and days to maturity where the observations recorded on plot basis.

\section{Results and Discussion}

Analysis of variance indicated significant difference among the genotypes for all the traits (Table-1). This indicates that there was 
an ample scope for selection of promising lines from the present gene pool for yield and its component traits. The presence of large amount of variability might be due to diverse source of materials taken as well as environmental influence affecting the phenotypes. These findings of mean sum of squares are in accordance with the earlier findings where significant variability for yield and its components in Black gram was observed.

\section{Mean performances of the different quantitative characters}

The mean values, the coefficient of variation (C.V.), standard error of the mean (SEm \pm ), the critical difference (C.D.) at $5 \%$ and $1 \%$, range of 30 genotypes for 13 quantitative characters are presented in Table- 2 which revealed a wide range of variation for all traits studied.

The mean performance of days to $50 \%$ flowering ranged from 35.66 days to 41.33 days with a grand mean of 38.6 days. The genotype SU-URD-101(35.66days), LBG20(35.66days) was earliest in terms of days to $50 \%$ flowering followed bySU-URD104(36.33days), IU-02-1-3(36.33days) and KPU-13-192(36.54days). The genotype SUURD-108(41.33days) took maximum days for $50 \%$ flowering followed by the genotypes SHEKHAR-2 (C) (40.66days), NDUK-136(40.66days), NDUK-13-4(40.66days) and LPG-791(40.66days).

The mean performance of seed yield per plant ranged from 4.16 to $5.08 \mathrm{~g}$ with a grand mean of $4.6 \mathrm{~g}$. The genotype SU-URD-102(5.08g) recorded the highest seed yield per plant followed by the genotypes SU-URD-112 (5.02g), SU-URD-95 (4.96g), SU-URD-101 (4.95g) and SU-URD-106 (4.92g).All these genotypes were significantly higher when compared with check shekhar-2(4.64g).
Among these genotypes, genotype SU-URD101was found with high yield along with early flowering. It was found good for yield contributing traits also. Hence, genotype SUURD-101may be selected for earliness along with high yield. The genotypes SU-URD-101 may also be used in hybridization programme with the contrast parent for the selection of superior genotypes in segregating generations. The other high yielding genotypes may be recommended for cultivation in different maturity segments for higher production. The genotype SU-URD-103 (46 days) was earliest in terms of $50 \%$ pod setting followed bySUURD-97 (46.33days), SU-URD-104 (47.33days), SU-URD-113(47.33days) and SU-URD-101(47.66days). The genotype SUURD-102(61.66days) was earliest in terms of days to maturity followed bySU-URD108(61.66days), L-20(62days), NDUK-136(62.33days) and SU-URD-111(62.66days). The genotype SU-URD-96(47.3cm) was the tallest in terms of plant height followed bySU-URD-112 $(47.3 \mathrm{~cm}), \mathrm{UG}-27(47.26 \mathrm{~cm})$, SU-URD-107 $(47.26 \mathrm{~cm})$ and SU-URD$98(46.73 \mathrm{~cm})$. The genotype KPU-13-192 (2.94) has maximum number of primary branches per plant followed by the genotypes SU-URD-96(2.93), UG-27(2.93), SU-URD109(2.93) and SU-URD-101(2.93). The genotype SU-URD-96(11.6) has maximum number of clusters per plant followed by the genotypes SU-URD-103(11.4), IU-02-13(11.26), NDUK-13-6(11) and SU-URD99(10.93). The genotype SU-URD-96(31.66) has maximum number of pods per plant followed by the genotypes SU-URD-95(31.4), SU-URD-99(30.13), NDUK-13-6(28.1) and SU-URD-97(28). The genotype SU-URD$103(3.93 \mathrm{~cm})$ had highest pod length followed by the genotype SU-URD-107 $(3.91 \mathrm{~cm})$, LPG-791(3.9cm), SU-URD-111 $(3.9 \mathrm{~cm})$ and SU-URD-105(3.9cm). The genotype SUURD-98(6), UG-27(6), SU-URD-105(6) and SU-URD-107(6) has maximum number of seeds per pod followed by the genotypes SU- 
URD-103(5.66). VBD-11-016(22.62g) has highest biological yield per plant followed by the genotypes SU-URD-96 (22.42g), SUURD-110(21.82g), KPU-13-192 (21.56g) and SU-URD-103 (21.54g). The genotype SUURD-98(27.53\%) has highest harvest index followed by the genotypes SU-URD102(27.43\%), SU-URD-100(27.1\%), SUURD-03(27.02\%) and LBG-20(26.87\%). The genotype SU-URD-109 (3.66g) had maximum 100-seed weight followed by the genotypes SU-URD-103 (3.66g), SU-URD108(3.63g), NDUK-13-6(3.63g) and SUURD-97(3.62g).

In the present investigation, as expected, the PCV estimates were higher than the GCV estimates (table-3). Among the 13 quantitative characters GCV ranged from 2.5 for days to maturity to $11.32 \%$ (no. of clusters per plant) indicating considerable amount of variability present among the genotypes. Among all the characters, moderate GCV and PCV were observed fornumber of clusters per plant, harvest index $\%$ and number of pods per plant in comparison to other characters indicating the presence of medium amount of genetic variability for these characters. Selection for these characters would be effective because the response to selection is directly proportional to the variability present in the experimental material. Similar reports are reported by Narasimhulu et al., (2013), Kumar et al., (2015) and Hemlatha et al., (2017).

High heritability (broad sense) (>60\%) was recorded for character number of clusters per plant (95.3\%), number of pods per plant (94.4\%), harvest index \% (92.4\%), biological yield per plant (92\%), number of seeds/pod $(87.5 \%)$, number of primary branch $(85.5 \%)$, seed yield per plant $(83.4 \%), 100$-seed weight (g) $(70.8 \%)$ and days to $50 \%$ flowering $(68.6 \%)$. In the present investigation, high heritability along with moderate $\mathrm{gcv}$ was recorded for number of clusters per plant, harvest index $\%$ and number of pods per plant. This indicates closeness of respective $\sigma 2 p$ and $\sigma 2 \mathrm{~g}$ value thereby low environmental effect on expression of these characters. Thus phenotypic selection may be effective for these characters. Similar results are reported by Narasimhulu et al., (2013), Kumar et al., (2015) and Hemlatha et al., (2017).

Thus, based on the present study, characters like number of clusters per plant, harvest index $\%$ and number of pods per plant have high heritability along with GCV indicating that these traits were comparatively less affected by environments, hence desirable for selection in breeding programme. Johnson et al., (1955) suggested that without genetic advance the estimates of heritability will not be of practical value and emphasized the concurrent use of genetic advance along with heritability. High genetic advance as \% mean $(>20 \%)$ was recorded for number of clusters per plant, number of pods per plant, and harvest index $\%$ along with high heritability and moderate GCV. Such values may be attributed to the additive gene effects and direct selection for these traits would be fruitful. Similar results are reported by Narasimhulu et al, (2013), Kumar et.al. (2015), Gowsalya et al., (2016) and Kuralarasan et al., (2018).

\section{Correlation coefficient}

In general, genotypic correlation was higher than the phenotypic ones. This indicated an inherent association between various traits. In the present investigation, phenotypic and genotypic correlation coefficient analysis revealed that seed yield per plant exhibited positive and significant correlation with harvest index \% only. Seed yield per plant exhibited positive and non significant correlation with biological yield per plant, plant height $(\mathrm{cm})$, number of clusters per 
plant, number of primary branch. Seed yield per plant exhibited negative and significant correlation with plant days to $50 \%$ flowering and100-seed weight (g). Seed yield per plant exhibited negative and non significant correlation with number of seeds/pod, days to $50 \%$ pod setting and pod length $(\mathrm{cm})$ (Table $3 \& 4)$.

Significant positive association of theses above attributes indicated that these attributes were mainly influencing the grain yield in black gram. Thus, selection practiced for the improvement in one character will automatically result in the improvement of other character even though direct selection for improvement has not been made for the yield character. Similar results exhibiting highly significant and positive correlation between grain yield and other traits as obtained in the present investigation were in accordance with earlier findings of Islam et al., (1999) for plant height, and number of pods/plant, Gill et al., (2000) for number of pods/plant, Rajan et al., (2000)for Number of pods per plant, and harvest index, Dikshit et al., (2002) for biological yield, and harvest index, Rao et al., (2006) for number of pods per plant, biological yield per plant and harvest index, Parameshwarappa and Salimath (2007) for Pods per plant and plant height, Saxena et al., (2007) for pods per plant, biological yield per plant, and harvest index at both phenotypic and genotypic level, Achakzai et al, (2007) for number of pods plant, Konda et al., (2008) reported for pods per plant, had positive and highly significant genotypic and phenotypic correlation with seed yield.

The significant correlation at both the levels between above component characters can be used for simultaneous improvement in both the characters with selection for one character only while selection for correlated character may not be done. However, significant correlation only at genotypic level reflects the masking effects of the environment (Table 57).

Table.1 Mean Sum of Squares for different characters in Black gram (Zaid, 2019)

\begin{tabular}{|c|l|c|c|c|}
\hline S. No & Character & \multicolumn{3}{|c|}{ Mean Sum of Squares } \\
\cline { 3 - 5 } & & $\begin{array}{c}\text { Replication } \\
(\mathbf{d . f = 2})\end{array}$ & $\begin{array}{c}\text { Treatment } \\
(\mathbf{d . f}=\mathbf{2 9})\end{array}$ & $\begin{array}{c}\text { Error } \\
(\mathbf{d} . \mathbf{f}=\mathbf{5 8})\end{array}$ \\
\hline $\mathbf{1}$ & Days to 50\% flowering & 0.696 & $8.789^{* *}$ & 1.165 \\
\hline $\mathbf{2}$ & Days to 50\% pod setting & 0.525 & $6.847^{* *}$ & 1.651 \\
\hline $\mathbf{3}$ & Days to maturity & 5.125 & $10.219^{* *}$ & 2.339 \\
\hline $\mathbf{4}$ & Plant height (cm) & 1.131 & $6.327^{* *}$ & 1.636 \\
\hline $\mathbf{5}$ & Number of primary branch & 0.006 & $0.093^{* *}$ & 0.005 \\
\hline $\mathbf{6}$ & Number of clusters per plant & $0.258^{*}$ & $3.559^{* *}$ & 0.058 \\
\hline $\mathbf{7}$ & Number of pods per plant & 0.951 & $20.559^{* *}$ & 0.401 \\
\hline $\mathbf{8}$ & Pod length(cm) & 0.006 & $0.041^{* *}$ & 0.009 \\
\hline $\mathbf{9}$ & Number of seeds/pod & 0.043 & $0.427 * *$ & 0.019 \\
\hline $\mathbf{1 0}$ & Biological yield per plant & 0.052 & $9.117^{* *}$ & 0.258 \\
\hline $\mathbf{1 1}$ & Harvest index \% & 0.011 & $18.325^{* *}$ & 0.486 \\
\hline $\mathbf{1 2}$ & 100-seed weight (g) & 0.013 & $0.053^{* *}$ & 0.006 \\
\hline $\mathbf{1 3}$ & Seed yield per plant & 0.008 & $0.220^{* *}$ & 0.014 \\
\hline
\end{tabular}


Table.2 Mean performance of thirty genotypes for 13 characters in black gram

\begin{tabular}{|c|c|c|c|c|c|c|c|c|c|c|c|c|c|}
\hline Genotypes & DF $50 \%$ & PS 50\% & DM & PH & $\mathbf{P B} / \mathbf{P}$ & $\mathbf{C} / \mathbf{P}$ & Pods/ P & PL & Sd/ Pods & BY & HI & SI & SYPP \\
\hline SU-URD-95 & 37.66 & 49.00 & 67.66 & 43.46 & 2.93 & 8.93 & 31.40 & 3.75 & 5.66 & 20.50 & 24.20 & 3.45 & 4.96 \\
\hline SU-URD-96 & 39.66 & 51.00 & 66.33 & 47.30 & 2.93 & 11.60 & 31.66 & 3.80 & 5.33 & 22.42 & 19.94 & 3.42 & 4.47 \\
\hline SU-URD-97 & 38.66 & 46.33 & 65.33 & 46.00 & 2.73 & 9.86 & 28.00 & 3.74 & 5.66 & 17.79 & 26.58 & 3.62 & 4.72 \\
\hline SU-URD-98 & 40.00 & 51.66 & 63.33 & 46.73 & 2.66 & 9.76 & 26.13 & 3.71 & 6.00 & 16.73 & 27.53 & 3.54 & 4.54 \\
\hline SU-URD-99 & 39.33 & 50.00 & 63.00 & 44.26 & 2.40 & 10.93 & 30.13 & 3.87 & 5.66 & 19.85 & 23.22 & 3.12 & 4.61 \\
\hline SU-URD-100 & 37.66 & 49.00 & 67.33 & 45.93 & 2.86 & 9.06 & 26.53 & 3.59 & 4.66 & 17.23 & 27.10 & 3.54 & 4.67 \\
\hline SU-URD-101 & 35.66 & 47.66 & 64.33 & 45.13 & 2.93 & 9.60 & 20.93 & 3.83 & 5.66 & 19.51 & 25.37 & 3.45 & 4.95 \\
\hline SU-URD-102 & 37.33 & 50.33 & 61.66 & 45.13 & 2.73 & 10.73 & 27.33 & 3.56 & 5.00 & 18.52 & 27.43 & 3.51 & 5.08 \\
\hline SU-URD-103 & 40.00 & 46.00 & 65.33 & 44.24 & 2.50 & 11.40 & 24.13 & 3.93 & 5.66 & 21.54 & 21.45 & 3.66 & 4.62 \\
\hline SU-URD-104 & 36.33 & 47.33 & 64.00 & 43.50 & 2.48 & 8.54 & 21.66 & 3.60 & 5.33 & 19.88 & 22.84 & 3.54 & 4.54 \\
\hline SU-URD-105 & 37.33 & 49.66 & 67.33 & 45.60 & 2.86 & 9.26 & 24.66 & 3.90 & 6.00 & 18.15 & 24.63 & 3.39 & 4.47 \\
\hline SU-URD-106 & 36.66 & 48.00 & 66.00 & 44.40 & 2.80 & 9.93 & 26.86 & 3.78 & 5.66 & 19.96 & 24.65 & 3.28 & 4.92 \\
\hline SU-URD-107 & 39.66 & 49.33 & 63.33 & 47.26 & 2.53 & 8.06 & 25.40 & 3.91 & 6.00 & 18.42 & 22.58 & 3.53 & 4.16 \\
\hline SU-URD-108 & 41.33 & 51.66 & 61.66 & 42.33 & 2.60 & 8.40 & 22.40 & 3.74 & 5.33 & 17.35 & 25.01 & 3.63 & 4.34 \\
\hline SU-URD-109 & 40.00 & 47.66 & 66.66 & 46.33 & 2.93 & 7.93 & 24.46 & 3.74 & 5.00 & 17.80 & 23.37 & 3.66 & 4.16 \\
\hline SU-URD-110 & 39.66 & 49.00 & 67.66 & 44.60 & 2.66 & 8.13 & 24.40 & 3.72 & 5.00 & 21.82 & 19.16 & 3.53 & 4.18 \\
\hline SU-URD-111 & 40.66 & 50.33 & 62.66 & 43.66 & 2.60 & 8.80 & 26.60 & 3.90 & 5.00 & 20.81 & 21.86 & 3.57 & 4.55 \\
\hline SU-URD-112 & 38.33 & 48.00 & 65.33 & 47.30 & 2.56 & 10.33 & 23.66 & 3.52 & 5.33 & 19.74 & 25.43 & 3.43 & 5.02 \\
\hline SU-URD-113 & 37.66 & 47.33 & 64.39 & 42.53 & 2.49 & 9.39 & 24.64 & 3.66 & 5.44 & 18.54 & 24.60 & 3.61 & 4.56 \\
\hline KPU-13-192 & 36.54 & 48.66 & 65.66 & 46.32 & 2.94 & 8.70 & 25.54 & 3.62 & 5.34 & 21.56 & 22.36 & 3.33 & 4.82 \\
\hline LPG-791 & 40.66 & 52.00 & 62.66 & 43.66 & 2.60 & 8.80 & 26.60 & 3.90 & 5.00 & 20.81 & 21.86 & 3.57 & 4.55 \\
\hline VBD-11-016 & 39.66 & 48.66 & 64.00 & 46.13 & 2.40 & 10.73 & 26.46 & 3.82 & 5.66 & 22.62 & 18.88 & 3.51 & 4.27 \\
\hline L-20 & 38.66 & 49.33 & 62.00 & 43.40 & 2.86 & 8.83 & 25.73 & 3.85 & 5.33 & 17.67 & 26.49 & 3.24 & 4.68 \\
\hline IU-02-1-3 & 36.33 & 47.66 & 66.33 & 45.53 & 2.80 & 11.26 & 26.20 & 3.56 & 5.33 & 18.61 & 22.41 & 3.47 & 4.17 \\
\hline NDUK-13-4 & 40.66 & 48.66 & 66.33 & 44.73 & 2.53 & 8.20 & 26.53 & 3.70 & 4.66 & 17.30 & 26.82 & 3.55 & 4.64 \\
\hline NDUK-13-6 & 40.66 & 50.33 & 62.33 & 43.73 & 2.66 & 11.00 & 28.10 & 3.75 & 5.66 & 17.35 & 24.03 & 3.63 & 4.17 \\
\hline UG-27 & 36.66 & 49.00 & 64.66 & 47.26 & 2.93 & 9.06 & 27.33 & 3.84 & 6.00 & 20.47 & 23.60 & 3.26 & 4.83 \\
\hline LBG-20 & 35.66 & 48.33 & 66.00 & 46.44 & 2.54 & 9.60 & 20.93 & 3.71 & 5.00 & 18.16 & 26.87 & 3.45 & 4.88 \\
\hline SU-URD-03 & 38.33 & 47.66 & 65.33 & 46.00 & 2.85 & 10.66 & 23.66 & 3.85 & 5.66 & 17.32 & 27.02 & 3.57 & 4.68 \\
\hline SHEKHAR-2 (C) & 40.66 & 49.66 & 66.33 & 44.73 & 2.60 & 8.80 & 26.53 & 3.70 & 5.33 & 17.30 & 26.82 & 3.55 & 4.64 \\
\hline Mean & 38.60 & 48.98 & 64.83 & 45.12 & 2.70 & 9.54 & 25.82 & 3.75 & 5.41 & 19.19 & 24.14 & 3.49 & 4.60 \\
\hline C.V. & 2.80 & 2.62 & 2.36 & 2.83 & 2.61 & 2.53 & 2.45 & 2.52 & 2.57 & 2.65 & 2.89 & 2.28 & 2.54 \\
\hline F ratio & 7.54 & 4.15 & 4.37 & 3.87 & 18.76 & 61.18 & 51.24 & 4.56 & 22.06 & 35.29 & 37.73 & 8.29 & 16.11 \\
\hline F Prob. & 0.00 & 0.00 & 0.00 & 0.00 & 0.00 & 0.00 & 0.00 & 0.00 & 0.00 & 0.00 & 0.00 & 0.00 & 0.00 \\
\hline S.E. & 0.62 & 0.74 & 0.88 & 0.74 & 0.04 & 0.14 & 0.37 & 0.05 & 0.08 & 0.29 & 0.40 & 0.05 & 0.07 \\
\hline C.D. 5\% & 1.76 & 2.10 & 2.50 & 2.09 & 0.12 & 0.39 & 1.04 & 0.15 & 0.23 & 0.83 & 1.14 & 0.13 & 0.19 \\
\hline C.D. $1 \%$ & 2.35 & 2.79 & 3.33 & 2.78 & 0.15 & 0.52 & 1.38 & 0.21 & 0.30 & 1.11 & 1.52 & 0.17 & 0.25 \\
\hline Range Lowest & 35.66 & 46.00 & 61.66 & 42.33 & 2.40 & 7.93 & 20.93 & 3.52 & 4.66 & 16.73 & 18.88 & 3.12 & 4.16 \\
\hline Range Highest & 41.33 & 52.00 & 67.66 & 47.30 & 2.94 & 11.60 & 31.66 & 3.93 & 6.00 & 22.62 & 27.53 & 3.66 & 5.08 \\
\hline
\end{tabular}

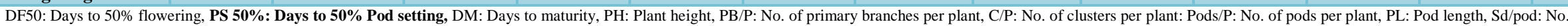

of seeds per pod, BY: Biological yield per plant, HI: Harvest index, SI: Hundred seed weight, SYPP: Seed yield per plant 
Table.3 Genetic parameters for 13 different characters in black gram (Zaid-2019)

\begin{tabular}{|l|c|c|c|c|c|}
\hline Characters & GCV & PCV & Heritability & GA & GA as \% mean \\
\hline Days to 50\% flowering & 4.130 & 4.987 & 68.6 & 2.719 & 7.044 \\
\hline Days to 50\% pod setting & 2.687 & 3.755 & 51.2 & 1.940 & 3.961 \\
\hline Days to maturity & 2.500 & 3.437 & 52.9 & 2.428 & 3.746 \\
\hline Plant height (cm) & 2.772 & 3.964 & 48.9 & 1.801 & 3.991 \\
\hline Number of primary branch & 6.357 & 6.873 & 85.5 & 0.327 & 12.112 \\
\hline Number of clusters per plant & 11.320 & 11.598 & 95.3 & 2.172 & 22.758 \\
\hline Number of pods per plant & 10.039 & 10.335 & 94.4 & 5.187 & 20.090 \\
\hline Pod length(cm) & 2.744 & 3.724 & 54.3 & 0.156 & 4.166 \\
\hline Number of seeds/pod & 6.812 & 7.281 & 87.5 & 0.711 & 13.129 \\
\hline Biological yield per plant & 8.954 & 9.338 & 92.0 & 3.394 & 17.688 \\
\hline Harvest index \% & 10.103 & 10.507 & 92.4 & 4.830 & 20.011 \\
\hline 100-seed weight (g) & 3.561 & 4.231 & 70.8 & 0.215 & 6.174 \\
\hline Seed yield per plant & 5.712 & 6.253 & 83.4 & 0.494 & 10.748 \\
\hline
\end{tabular}

Table.4 Phenotypic Correlation coefficient for different characters in black gram

\begin{tabular}{|c|c|c|c|c|c|c|c|c|c|c|c|c|}
\hline & DF $50 \%$ & PS 50\% & DM & PH & $\mathbf{P B} / \mathbf{P}$ & $\mathbf{C} / \mathbf{P}$ & Pods/ P & PL & Sd/ Pods & BY & HI & SI \\
\hline DF $50 \%$ & 1 & & & & & & & & & & & \\
\hline PS 50\% & $0.3239 * *$ & 1 & & & & & & & & & & \\
\hline DM & -0.1701 & $-0.2235 *$ & 1 & & & & & & & & & \\
\hline PH & -0.1162 & -0.126 & 0.206 & 1 & & & & & & & & \\
\hline PB/P & $-0.3059 * *$ & 0.0015 & $0.3171 * *$ & 0.2047 & 1 & & & & & & & \\
\hline $\mathbf{C} / \mathbf{P}$ & -0.0912 & -0.0821 & -0.0861 & 0.1727 & -0.0685 & 1 & & & & & & \\
\hline Pods/P & $0.2496 *$ & $0.2883 * *$ & 0.0902 & 0.0515 & 0.1681 & $0.2826 * *$ & 1 & & & & & \\
\hline PL & $0.2381 *$ & 0.1599 & -0.0862 & -0.1016 & -0.0167 & -0.0255 & 0.1341 & 1 & & & & \\
\hline Sd/Pods & -0.1072 & -0.0591 & -0.1454 & 0.1479 & 0.0717 & $0.2521 *$ & 0.0896 & $0.3289 * *$ & 1 & & & \\
\hline BY & -0.0647 & -0.0579 & 0.0787 & 0.0889 & -0.0564 & 0.1925 & 0.207 & 0.1747 & 0.0387 & 1 & & \\
\hline HI & -0.155 & -0.0273 & -0.0647 & -0.0011 & 0.1545 & -0.0943 & -0.1634 & $-0.2492 *$ & -0.0537 & $-0.7808 * * *$ & 1 & \\
\hline SI & $0.3840 * * *$ & -0.0547 & -0.0107 & -0.128 & -0.1995 & -0.1238 & $-0.2404 *$ & -0.0382 & $-0.2141 *$ & $-0.2412 *$ & 0.0065 & 1 \\
\hline SYPP & $-0.4364 * * *$ & -0.1461 & -0.0243 & 0.0414 & 0.2062 & 0.0962 & -0.005 & -0.2039 & -0.0163 & 0.0366 & $0.4786 * *$ & $-0.3224 * *$ \\
\hline
\end{tabular}


Table.5 Genotypic correlation coefficient for different characters in black gram

\begin{tabular}{|c|c|c|c|c|c|c|c|c|c|c|c|c|}
\hline & DF $50 \%$ & PS $50 \%$ & DM & PH & $\mathbf{P B} / \mathbf{P}$ & $\mathbf{C} / \mathbf{P}$ & Pods/ P & PL & Sd/ Pods & BY & HI & SI \\
\hline DF $50 \%$ & 1 & & & & & & & & & & & \\
\hline PS $50 \%$ & $0.589 * *$ & 1 & & & & & & & & & & \\
\hline DM & $-0.378 * *$ & $-0.637 * *$ & 1 & & & & & & & & & \\
\hline $\mathbf{P B} / \mathbf{P}$ & $-0.429 * *$ & -0.024 & $0.400 * *$ & $0.342 * *$ & 1 & & & & & & & \\
\hline $\mathbf{C} / \mathrm{P}$ & -0.125 & -0.129 & -0.099 & 0.186 & -0.0574 & 1 & & & & & & \\
\hline Pods/P & $0.280^{*}$ & $0.378 * *$ & 0.036 & 0.045 & 0.186 & $0.302 *$ & 1 & & & & & \\
\hline Sd/Pods & -0.142 & -0.071 & -0.123 & 0.234 & 0.067 & $0.286^{*}$ & 0.116 & $0.509 * *$ & 1 & & & \\
\hline BY & -0.072 & -0.047 & 0.145 & 0.038 & -0.046 & 0.196 & 0.224 & $0.259 *$ & 0.046 & 1 & & \\
\hline HI & -0.22 & -0.023 & -0.076 & 0.016 & 0.154 & -0.088 & -0.179 & $-0.347 * *$ & -0.071 & $-0.858 * *$ & 1 & \\
\hline SI & $0.484 * *$ & -0.141 & -0.01 & -0.201 & $-0.259 *$ & -0.136 & $-0.300 *$ & -0.175 & $-0.285^{*}$ & $-0.263^{*}$ & 0.026 & 1 \\
\hline SYPP & $-0.562 * *$ & -0.184 & 0.088 & 0.083 & 0.227 & 0.119 & 0.009 & -0.201 & -0.045 & 0.037 & $0.540 * *$ & $-0.429 * *$ \\
\hline
\end{tabular}

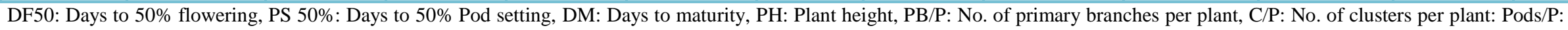

No. of pods per plant, PL: Pod length, Sd/pod: No. of seeds per pod, BY: Biological yield per plant, HI: Harvest index, SI: Hundred seed weight, SYPP: Seed yield per plant

* Significant at $\mathrm{P}=0.05$,** Significant at $\mathrm{P}=0.01$

Table.6 Phenotypic direct (diagonal) and indirect effects (non-diagonal) of different characters on seed yield per plant in black gram

\begin{tabular}{|c|c|c|c|c|c|c|c|c|c|c|c|c|}
\hline Traits & DF $50 \%$ & PS 50\% & DM & PH & $\mathbf{P B} / \mathbf{P}$ & $\mathbf{C} / \mathbf{P}$ & Pods/ P & PL & Sd/ Pods & BY & HI & SI \\
\hline DF $50 \%$ & -0.1809 & -0.0586 & 0.0308 & 0.021 & 0.0554 & 0.0165 & -0.0452 & -0.0431 & 0.0194 & 0.0117 & 0.028 & -0.0695 \\
\hline PS 50\% & -0.0114 & -0.0352 & 0.0079 & 0.0044 & -0.0001 & 0.0029 & -0.0101 & -0.0056 & 0.0021 & 0.002 & 0.001 & 0.0019 \\
\hline DM & 0.0122 & 0.0161 & -0.0719 & -0.0148 & -0.0228 & 0.0062 & -0.0065 & 0.0062 & 0.0105 & -0.0057 & 0.0047 & 0.0008 \\
\hline PH & 0.0082 & 0.0089 & -0.0145 & -0.0703 & -0.0144 & -0.0121 & -0.0036 & 0.0071 & -0.0104 & -0.0062 & 0.0001 & 0.009 \\
\hline $\mathrm{PB} / \mathrm{P}$ & -0.0142 & 0.0001 & 0.0148 & 0.0095 & 0.0466 & -0.0032 & 0.0078 & -0.0008 & 0.0033 & -0.0026 & 0.0072 & -0.0093 \\
\hline $\mathbf{C} / \mathbf{P}$ & 0.0001 & 0.0001 & 0.0001 & -0.0002 & 0.0001 & -0.0011 & -0.0003 & 0 & -0.0003 & -0.0002 & 0.0001 & 0.0001 \\
\hline Pods/P & 0.0123 & 0.0142 & 0.0044 & 0.0025 & 0.0083 & 0.0139 & 0.0492 & 0.0066 & 0.0044 & 0.0102 & -0.008 & -0.0118 \\
\hline PL & -0.0108 & -0.0072 & 0.0039 & 0.0046 & 0.0008 & 0.0012 & -0.0061 & -0.0452 & -0.0149 & -0.0079 & 0.0113 & 0.0017 \\
\hline Sd/Pods & 0.0011 & 0.0006 & 0.0015 & -0.0015 & -0.0007 & -0.0025 & -0.0009 & -0.0033 & -0.0101 & -0.0004 & 0.0005 & 0.0022 \\
\hline BY & -0.061 & -0.0546 & 0.0742 & 0.0837 & -0.0531 & 0.1814 & 0.1951 & 0.1646 & 0.0364 & 0.9422 & -0.7357 & -0.2273 \\
\hline HI & -0.1813 & -0.0319 & -0.0757 & -0.0013 & 0.1807 & -0.1103 & -0.1911 & -0.2915 & -0.0628 & -0.9132 & 1.1696 & 0.0076 \\
\hline SI & -0.0107 & 0.0015 & 0.0003 & 0.0036 & 0.0056 & 0.0034 & 0.0067 & 0.0011 & 0.006 & 0.0067 & -0.0002 & -0.0278 \\
\hline SYPP & $-0.4364 * *$ & -0.1461 & -0.0243 & 0.0414 & 0.2062 & 0.0962 & -0.005 & -0.2039 & -0.0163 & 0.0366 & $0.4786^{* *}$ & $-0.3224 * *$ \\
\hline
\end{tabular}


Table.7 Genotypic direct (diagonal) and indirect effects (non-diagonal) of different characters on seed yield per plant in black gram

\begin{tabular}{|c|c|c|c|c|c|c|c|c|c|c|c|c|}
\hline Traits & DF $50 \%$ & PS $50 \%$ & DM & PH & $\mathbf{P B} / \mathbf{P}$ & $\mathrm{C} / \mathrm{P}$ & Pods/ P & PL & Sd/ Pods & BY & HI & SI \\
\hline DF $50 \%$ & -0.4303 & -0.2534 & 0.1625 & 0.1306 & 0.1848 & 0.0536 & -0.1203 & -0.183 & 0.0611 & 0.0311 & 0.0945 & -0.2081 \\
\hline PS 50\% & 0.1189 & 0.2019 & -0.1286 & -0.0165 & -0.0048 & -0.0261 & 0.0762 & 0.033 & -0.0144 & -0.0095 & -0.0047 & -0.0285 \\
\hline DM & -0.0517 & -0.0871 & 0.1368 & 0.0626 & 0.0548 & -0.0136 & 0.005 & -0.0427 & -0.0168 & 0.0198 & -0.0103 & -0.0014 \\
\hline PH & 0.0081 & 0.0022 & -0.0122 & -0.0266 & -0.0091 & -0.0049 & -0.0012 & 0.0025 & -0.0062 & -0.001 & -0.0004 & 0.0053 \\
\hline $\mathbf{P B} / \mathbf{P}$ & 0.0843 & 0.0046 & -0.0786 & -0.0671 & -0.1962 & 0.0113 & -0.0365 & 0.0068 & -0.0131 & 0.009 & -0.0303 & 0.0508 \\
\hline $\mathbf{C} / \mathbf{P}$ & 0.0054 & 0.0056 & 0.0043 & -0.0081 & 0.0025 & -0.0437 & -0.0132 & -0.0003 & -0.0125 & -0.0086 & 0.0039 & 0.0059 \\
\hline Pods/P & 0.0347 & 0.0469 & 0.0045 & 0.0056 & 0.0231 & 0.0375 & 0.1241 & 0.0141 & 0.0144 & 0.0278 & -0.0222 & -0.0372 \\
\hline PL & 0.1443 & 0.0554 & -0.1061 & -0.0314 & -0.0118 & 0.0026 & 0.0386 & 0.3394 & 0.1726 & 0.0878 & -0.1176 & -0.0594 \\
\hline Sd/Pods & 0.0135 & 0.0068 & 0.0118 & -0.0223 & -0.0063 & -0.0273 & -0.0111 & -0.0485 & -0.0954 & -0.0044 & 0.0068 & 0.0272 \\
\hline BY & -0.1293 & -0.084 & 0.2582 & 0.0676 & -0.0818 & 0.35 & 0.3994 & 0.462 & 0.0829 & 1.7856 & -1.5318 & -0.4697 \\
\hline SI & 0.1113 & -0.0324 & -0.0023 & -0.0462 & -0.0596 & -0.0313 & -0.069 & -0.0403 & -0.0655 & -0.0605 & 0.006 & 0.2301 \\
\hline SYPP & -0.5621 & -0.1837 & 0.0884 & 0.0833 & 0.2268 & 0.1188 & 0.0089 & -0.2008 & -0.0449 & 0.0365 & 0.5395 & -0.4286 \\
\hline
\end{tabular}




\section{Path coefficient analysis}

In the present investigation, both at phenotypic and genotypic level, the positive direct effect on grain yield was depicted by harvest index \%, biological yield per plant and number of pods per plant. At both the levels, the negative direct effect on grain yield was depicted by plant height $(\mathrm{cm})$, number of clusters per plant, number of seeds/pod, and days to $50 \%$ flowering. The residual component of phenotypic and genotypic path analysis indicated that 66 and $72 \%$ of variability of seed yield was accounted for by these thirteen characters.

Path analysis further revealed that at both phenotypic and genotypic levels, the positive and negative indirect effects through harvest index $\%$, biological yield per plant, number of pods per plant, plant height $(\mathrm{cm})$, number of clusters per plant, number of seeds/pod, and days to $50 \%$ flowering was observed at both the level.

Similar results have been reported by previous workers. Rajan et al., (2000) reported that number of pods per plant had the highest positive direct effect on seed yield, followed by 100 -seed weight. Dikshit et al., (2002) reported that the harvest index exhibited maximum direct effect on seed yield per plant. Haritha and Sekhar (2002) reported that biological yield/plant exhibited maximum direct effect on grain yield. Hence selection based on these characters would bring an improvement in grain yield in Black gram. Hassan et al., (2003) observed great indirect effect via biological yield resulted in highly significant correlation with grain yield. Parameshwarappa and Salimath (2007) reportad that pods per plant had the highest positive direct effect on yield per plant followed by test seed weight and number of branches. Saxena et al., (2007) revealed that an early maturing dwarf plant with high biological yield and harvest index would be suitable for higher seed yield in black gram. Prasanna et al., (2013) reported that the harvest index, number of seeds per pod and days to maturity recorded to have maximum positive direct effect towards seed yield per plant. Punia et al., (2013) Path coefficient analysis also revealed that number of pods per plant and number of branches per plant had major direct and indirect effect on yield contributing traits in black gram.

Path analysis further revealed that direct effect of harvest index \%, biological yield per plant and numbers of pods per plant were of high magnitude. The high positive association of other characters with grain yield per plant (g) was also due to high indirect effect through these characters. This indicated that grain yield was mainly a product of direct and indirect effects (through each other) of harvest index \%, biological yield per plant and number of pods per plant. Path analysis further revealed seed index was negatively associated with grain yield per plant $(\mathrm{g})$ which was due to the negative direct effects of seed index. Path analysis further revealed days to $50 \%$ flowering was negatively associated with grain yield per plant (g). Days to $50 \%$ flowering though have negative direct effects at both the level on grain yield but had negative association because of indirect effects through harvest index \%, biological yield per plant and number of pods per plant. This indicated that seed yield was mainly a product of direct and indirect effects of above three characters.

The results from the present investigation conclude that significant differences were recorded for all the thirteen characters among the 30 black gram genotypes included in the study, indicating presence of sufficient variation among them. The genotype SUURD-102(5.08), SU-URD-112 (5.02), SUURD-95 (4.96g), SU-URD-101(4.95g), SU- 
URD-106 (4.92g), and LBG-20(4.88g) recorded statistically significant higher yield when compared with the check variety SHEKHAR-2 (4.64g). Number of pods per plant and Harvest index \% had high genetic advance as \% mean along with high heritability and GCV. At phenotypic and genotypic levels, seed yield per plant exhibited positive and significant correlation with harvest index only. It had negative and significant correlation with days to $50 \%$ flowering and 100seed weight. At both the levels, highest positive direct effect on grain yield was depicted by harvest index and biological yield per plant indicating their importance in selection for yield improvement.

\section{References}

Achakzai, A. K. K. and Kamran Panizai M. (2007). Effect of row spacing on growth yield and yield components in mashbean. Sarhad Journal Agriculture, 23: $5-10$

Dikshit, H. K., Singh, B. B. and Dua, R. P. (2002).Genetic variation in mung bean. Indian, 15: 125-127.

Gill, J.S., Gumber, R.K., Verma, M.M. and Rathore, P. (2000). Genetic estimates of advanced black gram lines derived through different selection methods Crop improvement, 27 (1): 88-98.

Gowsalya, P, Kumaresan, D, Packiaraj, D and Kannan Bapu, J.R. (2016). Genetic variability and character association for biometrical traits in Blackgram (Vigna mungo (L.) Hepper). Vol 7 No 2 DOI: 10.5958/0975-928X.2016.00039.9

Haritha, S. and Sekhar, M. R. (2002). Correlation and path coefficient analysis in mung bean [Vigna radiata (L.) Wilczek] Legume Research, 25: 180-183.

Hemalatha, K, S.S. Lal and G.M. Lal. (2017). Study on Genetic Variability and
Correlation in Blackgram (Vigna mungoL. Hepper). Journal of Pharmacognosy and Phytochemistry 2017; 6(4): 674-676

Islam, M.T., Haque, M.M., Islam, M.O., Malick, M.A. and Haque, M.A. (1999).Genetic variability correlation and path analysis in mung bean (Vigna radiata L. Wilczek). Bangladesh Journal of Science Industrial Research, 34 (1): 103-107.

Johnson, H.W., Robinson, H.F. and Comstock, R.E. (1955). Genotypic and Phenotypic Correlations in Soybean and their implications in selection. Agronomy, 47(1): 477-438.

Konda, C.R., Salimath, P.M. and Mishra, M.N. (2008). Correlation and path coefficient analysis in black gram (Vigna mungo (L.) Hepper). Legume Research, 31(3): 202-205.

Kumar, G.V., Vanaja, M., Sathish, P., Vagheera, P. and Lakhsmi, N. J. (2015). Correlation analysis for quantitative traits in black gram (Vigna mungo (L.) Hepper) in different seasons International Journal of Scientific and Research Publications, 5 (4) 2250-3153

Kuralarasan, V., Vanniarajan, C, Kanchana, S, Veni, K and Anandhi Lavanya, S. (2018) Genetic divergence, heritability and genetic advance in mutant lines of urdbean [Vigna mungo (L.) Hepper]. Legume Research, Print ISSN: 02505371.

Narasimhulu, R., Naidu N.V., ShanthiPriya M., Rajarajeswari V. and Reddy K.H.P. (2013). Genetic variability and association studies for yield attributes in mung bean [Vigna radiata (L.) Wilczek]. Indian Journal of Plant Sciences, 2(3): 82-86.

Parameswarappa, S. G. and Salimath, P. M. (2007). Studies on genetic variability, character association and path 
coefficient analysis in green gram, Crop Research, 34: (1/3) 195-197.

Prasanna, B. L., Rao, P. J. M., Murthy, K. G. K. and Prakash, K. K. (2013). Genetic variability, correlation and path coefficient analysis in mungbean. Environment and Ecology, 31(4): 17821788.

Punia, S.S., Gautam, and Verma, B.R.N.K. (2014). Genetic variability and correlation studies in urdbean (Vigna mungo) Agricultural Research Communication Center, 580-584

Rajan, R. E. B., Wilson, D., Vijayaraghava, K. and Kumar, V. (2000). Correlation and path analysis in the $F_{2}$ generation of green gram [Vigna radiata (L.) Wilczek]. Madras Agriculture Journal, 87(2): 590-593.

Rao, C. M., Rao, Y. K. and Mohan, R. (2006).Genetic variability and path analysis. Legume Research, 29(3): 216218.

Saxena, R. R., Singh, P. K. and Saxena, R. R. (2007).Correlation and path analysis in mung bean cultivars [Vigna radiata (L.) Wilczek]. Journal of Interacademicia, 11(2): 143-148.

Wright, S. (1921). Correlation and causation. ournalj. Agriculture Research, 20: 557585.

\section{How to cite this article:}

Ankur Kumar Singh and Gaibriyal M. Lal. 2020. Genetic Variability and Path Analysis for Quantitative Characters in F4 Generation of Black Gram (Vigna mungo L. Hepper). Int.J.Curr.Microbiol.App.Sci. 9(12): 112-123. doi: https://doi.org/10.20546/ijcmas.2020.912.015 